\title{
Direction repulsion in unfiltered and ring-filtered Julesz textures
}

\author{
DELWIN T. LINDSEY \\ Ohio State University, Mansfield, Ohio
}

\begin{abstract}
Perceived directions of motion were measured for each of two superposed two-dimensional dynamic random patterns consisting of unfiltered or ring-filtered dense random-check (Julesz) textures. One pattern always moved in a cardinal direction (up, down, left, or right), and the other texture always moved in an oblique direction separated from the cardinal component by $20^{\circ}-80^{\circ}$. Several cardinal/oblique speed ratios were tested. In Experiment 1, the textures were unfiltered. In Experiment 2 , the textures were ring filtered and had the same center frequency $(1,2$, or $4 \mathrm{cpd})$. In Experiment 3, a 1-cpd ring-filtered texture was paired with a 2-, 4-, or 8-cpd texture. Subjects consistently misperceived the directions of component motion in these experiments; the angular separation of movement of the two textures was perceptually exaggerated, a phenomenon referred to as direction repulsion (Marshak \& Sekuler, 1979). The results show that (1) direction repulsion occurs across at least a fourfold range of spatial frequencies and a sixfold range of speed ratios, (2) direction repulsion varies systematically with speed ratio, and (3) across most conditions, direction repulsion is anisotropic-direction repulsion is more evident in the oblique directions than in the cardinal directions. These findings suggest that the spatiotemporal range of inhibitory interactions involved in motion transparency is much greater than previously appreciated.
\end{abstract}

Individual low-level motion sensors are not highly selective for the speeds and directions of object motion. As a result, an object undergoing, say, simple linear translation will excite many different populations of sensors that differ from one another in their spatiotemporal characteristics. Furthermore, the univariant response properties of any given class of motion sensor make it unsuitable for signaling simultaneously and unambiguously both object speed and object direction. Thus, some form of integration across multiple sensor types is necessary in the computation of object motion. Cooperative pooling could, in principle, recover an estimate of object velocity in this situation-say, by computing a weighted mean, mode, or similar estimate (e.g., Adelson \& Movshon, 1982; Mingolla, Todd, \& Norman, 1983; Watanabe \& Cole, 1995; Williams \& Sekuker, 1984; Zohary, Scase, $\&$ Braddick, 1996). A problem, however, arises when the velocities of objects must be recovered under conditions of motion transparency. Under these conditions, it is desirable to partition sensor responses into those originating from each moving component so that the individual object velocities can be computed. Cooperative processes alone are not well suited for this task. Therefore, several computationalapproaches to motion transparency use some form of inhibitory, suppressive, and/or competitive process, in addition to excitatory, cooperative processes, in an attempt to recover the individual sources

This work was supported by Grant SRB-9514522 to the author from the National Science Foundation. Correspondence concerning this article should be addressed to D. T. Lindsey, Department of Psychology, Ohio State University, Mansfield, OH 44906 (e-mail: lindsey.43@ osu.edu). of transparent motion (e.g., Nowlan \& Sejnowski, 1995; Simoncelli \& Heeger, 1998; Wilson \& Kim, 1994).

Inhibitory interactions in motion processing have been revealed psychophysically by a number of phenomena, including motion cancellation and direction repulsion. Motion cancellation refers to the apparent reduction in sensitivity to either of two motion signals when both are presented in transparent motion, as compared with when either is presented alone. Motion cancellation appears to be greatest when the two motion signals differ from one another in direction by $180^{\circ}$. A classical example of motion cancellation is the absence of motion in counterphasemodulated cosine gratings (e.g., Stromeyer, Kronauer, Madsen, \& Klein, 1984). Another example of motion cancellation comes from experiments involving randomcheck patterns moving in dynamic noise. Motion is less detectable when the check patterns move in opposite directions than when only a single moving check pattern is present (Lindsey \& Todd, 1998; Mather \& Moulden, 1983; Verstraten, Fredericksen, van Wezel, Boulton, \& van de Grind, 1996; see also, Qian, Andersen, \& Adelson, 1994a). Similar effects have been reported also for superposed patterns moving in orthogonal directions (Snowden, 1989, 1990). These phenomena correlate well with some of the properties of mechanisms located in area V5/MT (Qian \& Andersen, 1994; Snowden, Treue, Erikson, \& Anderson, 1991), a region of the primate brain thought to play an important role in motion perception.

Direction repulsion, another kind of motion phenomenon that has been associated with inhibition in motion processing, is the subject of the present study. Direction repulsion refers to an exaggeration of the difference be- 
tween the perceived directions of motion of two arrays of moving elements sliding transparently past one another. Direction repulsion is illustrated in Figure 1. Two dynamic random-dot arrays are shown moving at velocities indicated by the solid arrows. When subjects are asked to indicate the perceived direction of motion of one or the other array of moving elements, their responses are similar to those indicated by the dashed arrows in the figure. The earliest systematic study of this phenomenon was by Marshak and Sekuler (1979), using sparse random-dot arrays. Motion repulsion was maximal when the angular separation was $20^{\circ}$ and declined for smaller or larger angles. Direction repulsion has been reported in a number of other studies involving not only dynamic random-dot arrays (Hiris \& Blake, 1996; Mather \& Moulden, 1980; Snowden, 1989), but also moving grating stimuli (Kim \& Wilson, 1996; Lindsey \& Todd, 1996).

Although there are several qualitative and quantitative models of direction repulsion that incorporate inhibitory interactions (e.g., Giese, 1999; Hiris \& Blake, 1996; Wilson \& Kim, 1994), there has been surprisingly little empirical work on these interactions across spatial and temporal scales. In a study using plaid and multiaperture bar patterns, Kim and Wilson (1996) found that interactions leading to direction repulsion spanned a wide range of speed differences between components; these interactions appeared to be confined to the same spatial scale. However, these authors employed angular separations that exceeded $120^{\circ}$. Large angular separations of the components-typically, $90^{\circ}$ or more-were needed to sustain motion transparency when grating or multiaperture

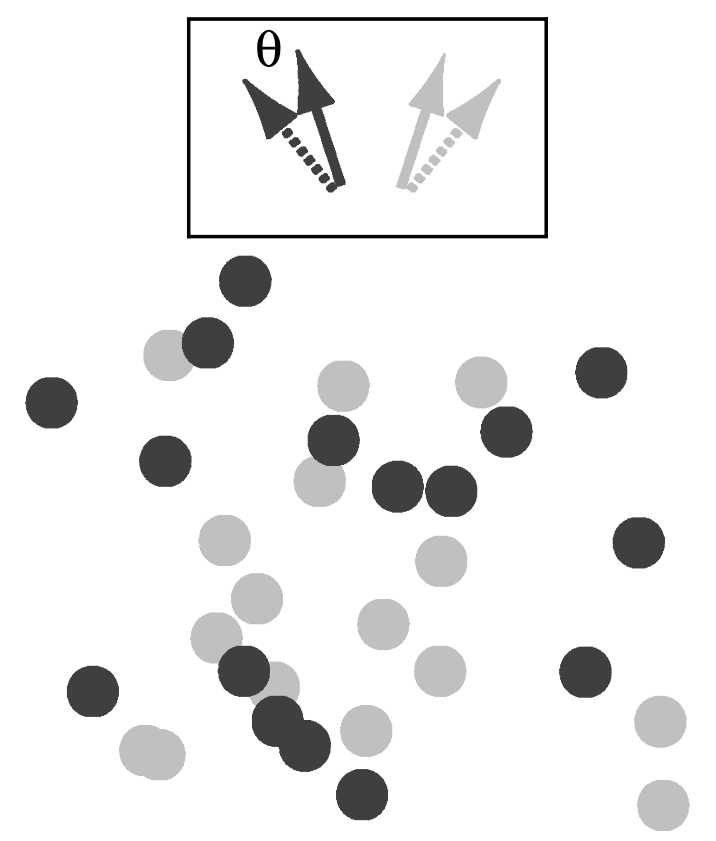

Figure 1. Direction repulsion $(\theta)$ is an exaggeration in perceived direction of motion (dashed arrows) of two superposed random-dot patterns (represented by light and dark dots, respectively) moving in different directions (solid arrows). bar stimuli were used (see Kim \& Wilson, 1993). On the other hand, other studies have shown that angular separations of $20^{\circ}-30^{\circ}$ sustain motion transparency in sparse random-dot patterns and lead to near-maximal direction repulsion (Hiris \& Blake, 1996; Marshak \& Sekuler, 1979). Small angular separations also sustain transparency in dynamic Julesz texture patterns (van Doorn \& Koenderink, 1983). There have been no empirical studies of interactions across spatial and temporal scales in this regime.

The principal goal of the present study was to examine interactions across different spatial and temporal scales, using spatially filtered or unfiltered dense random-check patterns, which I will call Julesz textures. In the unfiltered versions of these stimuli, each texture consisted of a twodimensional (2-D) array of elements. Each element specified an intensity value, drawn randomly (with replacement) from an equal-probability distribution of intensities. In one experiment, two moving unfiltered textures were additively combined in animation sequences that simulated two transparent textures moving in different directions and/or speeds. In this way, I studied direction repulsion in superposed textures that were spatially broadband, like the sparse random-dot patterns used previously by others. In two other experiments, the stimuli consisted of two ring-filtered Julesz textures (Chang \& Julesz, 1983; Cleary \& Braddick, 1990). Ring filtering was used to spatially bandpass filter the textures while leaving the orientation bandwidth of the textures unchanged. Thus, ring-filtered Julesz textures remained rich in 2-D spatial structure. Interactions within a single spatial scale were explored by applying identical filters to unfiltered Julesz patterns, whereas interactions across spatial scale were explored by applying filters with different passbands (identical octave band widths but different center frequencies) to the unfiltered textures.

\section{METHOD}

\section{Overview of Experimental Method}

In each of the three experiments described below, subjects sat in front of a computer display and binocularly viewed 1.5-sec-long animation sequences simulating two superposed dynamic randomtexture patterns presented within a $12.7^{\circ}$ circular aperture. In Experiment 1 , the two textures consisted of unfiltered Julesz patterns (see Figure 2a). In the second and third experiments (Figures $2 \mathrm{~b}$ and $2 \mathrm{c})$, the textures were ring-filtered Julesz textures. In Experiment 2, the two filtered textures had the same passband, whereas in Experiment 3, the textures had different passbands. With the exception of a series of control conditions run in Experiment 1, one of the textures always moved in a cardinal direction (up, down, left, or right), and the other texture always moved obliquely, in an intermediate direction (see Figure $2 \mathrm{~d}$ ). The subjects fixated a small red spot placed in the center of the stimulus display during each animation sequence. Following each stimulus presentation, two pointers, arranged like hands on a clock, replaced the textures in the stimulus display area of the monitor. The subjects used the computer mouse to adjust the direction of each pointer so that it corresponded to the perceived direction of motion of one of the previously viewed components in the stimulus. If both textures appeared to cohere and move in the same direction, the subjects adjusted both pointers to indicate the 

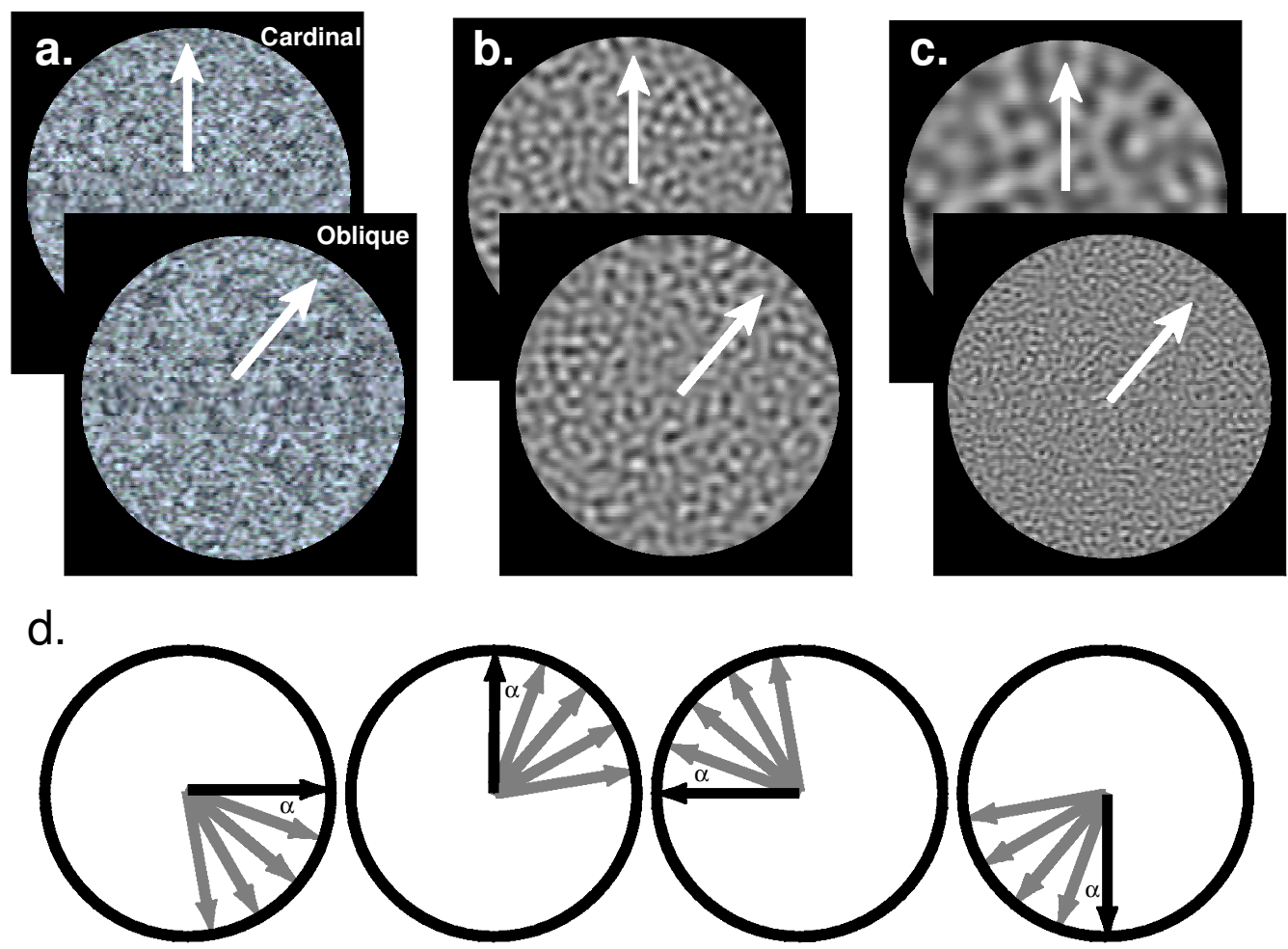

Figure 2. Overview of the stimuli used in this study. (a) In Experiment 1, the stimuli consisted of spatial superpositions of two unfiltered random-check textures (textures are shown separately in the figure for clarity). One texture moved in a cardinal direction while the other moved in an oblique direction. (b) In Experiment 2, the textures were ring filtered. Center frequencies of the two component textures were the same: 1,2 , or 4 cpd. (c) In Experiment 3, superposed ring-filtered textures had different center frequencies. A 1-cpd ring-filtered texture moving in a cardinal direction was paired with a 2-, 4-, or 8-cpd texture moving in an oblique direction. (d) In the main part of Experiment 1, one of four cardinal components—right, up, left, or down-was paired with an oblique component on each trial. The oblique component moved $20^{\circ}, 40^{\circ}, 6^{\circ}$, or $80^{\circ}$ clockwise from the cardinal component (e.g., $\alpha$ in panel d). In Experiments 2 and 3, the cardinal component was paired with $20^{\circ}, 4^{\circ}$, or $60^{\circ}$ oblique components. In the control part of Experiment 1, one texture remained stationary, and the other moved in one of the cardinal directions or in a direction $20^{\circ}, 40^{\circ}$, or $60^{\circ}$ clock wise from cardinal.

direction of coherent motion. When the series of control conditions was run, the same protocol was followed, except that only one of the components moved during the trial; the other component was stationary. The subjects adjusted only one pointer to indicate the direction of motion of the moving texture.

\section{Apparatus}

The animation sequences were generated on an SGI High Impact color graphics workstation (Silicon Graphics) and displayed on a 20 -in., high-resolution RGB monitor $(1,280 \times 1,024$ pixels, $\mathrm{h} \times \mathrm{v}$; $24 \mathrm{bits} /$ pixel; $60 \mathrm{~Hz}$, noninterlaced). Custom gamma correction based on calibration of the monitor's light output ensured that it was linear with signal input to within a few percent (see Lindsey \& Todd, 1998 , for a description of the monitor calibration procedures). At the viewing distance of $57.3 \mathrm{~cm}$, each pixel subtended $1.59 \mathrm{~min}$ arc of visual angle. A mean stimulus luminance of $97 \mathrm{~cd} / \mathrm{m}^{2}$ was maintained throughout the study. The animation sequences that produced the moving stimuli always occurred at a rate of 60 frames per second. The SGI workstation employs a double-buffering scheme. Thus, the transition from one image to the next in the animation sequence always occurred during the display's vertical-blanking interval.

\section{Stimuli}

The unfiltered Julesz textures consisted of 2-D arrays of achromatic elements, 2 pixels on each side $(3.18 \times 3.18$ arcmin $)$. Each element was drawn pseudorandomly (with replacement) from an equal-probability distribution of intensities on the interval $[0,127]$. Each stimulus was produced from two such arrays of intensities, stored in a special-purpose texture memory on board the workstation's graphics card. An example of the resulting textures is shown in Figure 2a. During animation, special-purpose hardware was used to map, to combine additively, and then to display on the computer monitor appropriate portions of each of these two texture arrays. Filtered textures were created by 2 -D convolution of $1,024 \times 1,024$ element unfiltered Julesz textures with appropriate ring filters. The point spread function, $g(r)$, of a particular ring filter was derived from products of Gaussian and Bessel functions (Watson \& Eckert, 1994):

$$
g(r)=J_{0}\left(2 \pi f_{c}|r|\right) e^{-\pi(|r| / s)^{2}},
$$

where

$$
s=\left(1 / f_{c}\right)\left(2^{b w}+1\right)\left(2^{b w}-1\right) \sqrt{\frac{\log 2}{\pi}},
$$

$r$ is the distance from the center of the kernel, $f_{c}$ is the desired center frequency of the ring filter, and $b w$ is the full-width, half-amplitude bandwidth (in octaves) of the filter. Spatial convolution was performed in the Fourier domain, using routines based on those published by Press, Teukolsky, Vetterling, and Flannery (1993). The result was then inverse-Fourier-transfor med back into the spatial 
domain to create the appropriately filtered textures. Following convolution, the intensities of the elements in the filtered patterns were scaled to give the maximum possible contrast. Samples of the textures used in Experiments 2 and 3, as well as average Fourier power spectra for these textures, are shown in Figure 3.

\section{Experimental Procedures}

In Experiment 1, each stimulus consisted of two superposed unfiltered dynamic random-check texture patterns. In a set of control measurements, one of the textures was stationary throughout the trial, and the other texture moved at $1.6 \mathrm{deg} / \mathrm{sec}$ in one of $16 \mathrm{di}$ rections, spanning $360^{\circ}$. The directions were the 4 cardinal directions and 12 oblique directions $\left(20^{\circ}, 40^{\circ}\right.$, and $60^{\circ}$ clockwise from each cardinal direction). In the main part of Experiment 1, both component textures moved. Perceived directions of the component motions were measured for 7 combinations of cardinal and oblique component speed drawn from a range of speeds from 0.27 to $1.6 \mathrm{deg} / \mathrm{sec}$. These spanned cardinal:oblique speed ratios of 1:6 to 6:1. For each particular combination of cardinal and oblique speeds, the subjects made settings for 16 combinations of cardinal and oblique directions, which are depicted in Figure 2d. Thus, there were 112 conditions ( 7 speed ratios $\times 16$ direction combinations) in the main part of Experiment 1. The subjects made 10 settings of perceived cardinal and oblique directions of motion for each of these conditions.

In Experiment 2, the textures consisted of ring-filtered patterns, with center frequencies of 1,2 , or 4 cycles per degree (cpd). Both moving patterns in the stimulus had the same center frequency. Three cardinal:oblique speed conditions were run- -0.27 versus 1.6 , 1.6 versus 1.6 , and 1.6 versus $0.27 \mathrm{deg} / \mathrm{sec}$-corresponding to three cardinal:oblique speed ratios-1:6 (SF), 1:1 (FF), and 6:1 (FS). For each speed combination, the perceived directions of cardinal and oblique component motion were measured for 12 combinations of cardinal and oblique directions: 4 cardinal directions (leftward, rightward, upward, and downward motion) $\times 3$ oblique directions $\left(20^{\circ}, 40^{\circ}\right.$, or $60^{\circ}$ clockwise from the cardinal component). Thus, there were 108 conditions in Experiment 2, and five trials were run per condition. Experiment 2 was designed to examine interactions within a given spatial scale.

The design of Experiment 3 was identical to that of Experiment 2, except that the center frequency of the cardinal-direction pattern was always $1 \mathrm{cpd}$ and the center frequency of the oblique-direction pattern was 2, 4, or $8 \mathrm{cpd}$. Experiment 3 was designed to examine interactions across spatial scale, as well as across speeds.

\section{Subjects}

The author and one other experienced psychophysical observer served as subjects in all of the experiments described below. One subject was emmetropic, and the other wore corrective lenses; both had normal visual acuity, and both gave informed consent after the procedures had been explained to them.

\section{RESULTS}

\section{Experiment 1}

The results of the control conditions, in which the subjects indicated the direction of motion of a single moving unfiltered Julesz texture, are shown in Figure 4. Subject D.T.L.'s data all fall very close to the diagonal line, indicating a close correspondence between actual and perceived directions of motion of a transparent texture on a stationary background texture. Subject J.T.T.'s data deviate slightly from the diagonal line. In general, these deviations are unsystematic, except for oblique directions at $20^{\circ}$ from a cardinal direction. In those cases, the average of J.T.T.'s settings is $25.75^{\circ}$, a bias of $5.75^{\circ}\left( \pm 2.5^{\circ}\right)$ away from the nearest cardinal direction.

Panels a-h in Figure 5 show the subjects' settings for conditions in which the cardinal component moved at a speed equal to, or greater than, that of the oblique component. The $1.6 \mathrm{deg} / \mathrm{sec}$ cardinal components were paired

\section{$1.0 \mathrm{cpd}$}

\section{$2.0 \mathrm{cpd}$}

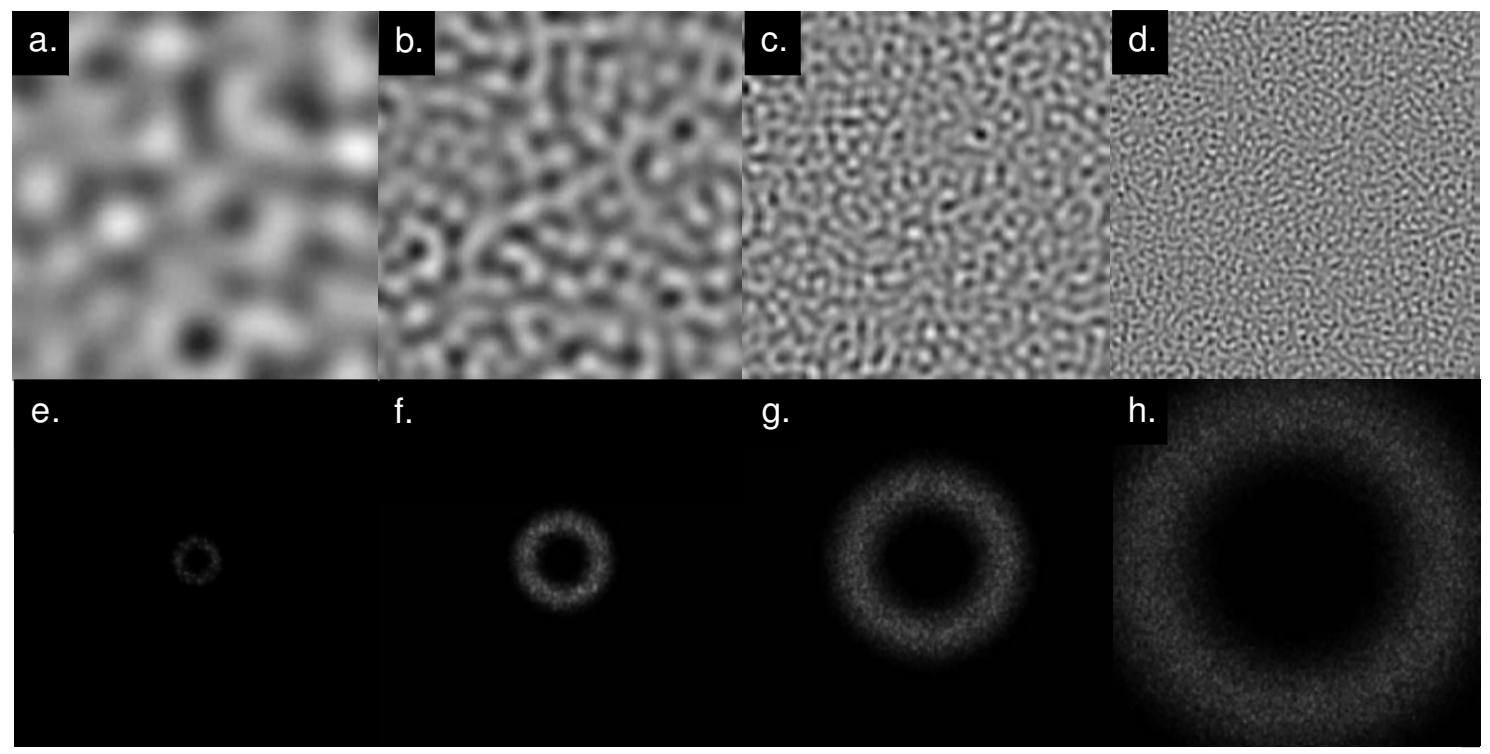

Figure 3. Ring-filtered textures used in Experiments 2 and 3. (a-d) Sample portions of textures produced by ring-filtering random-check patterns with kernels of center frequency $1,2,4$, and 8 cpd. The bandwidth of each filter was 1 octave (FWHH). (e-f) Average two-dimensional Fourier power spectra of textures like those depicted in panels a-d. 

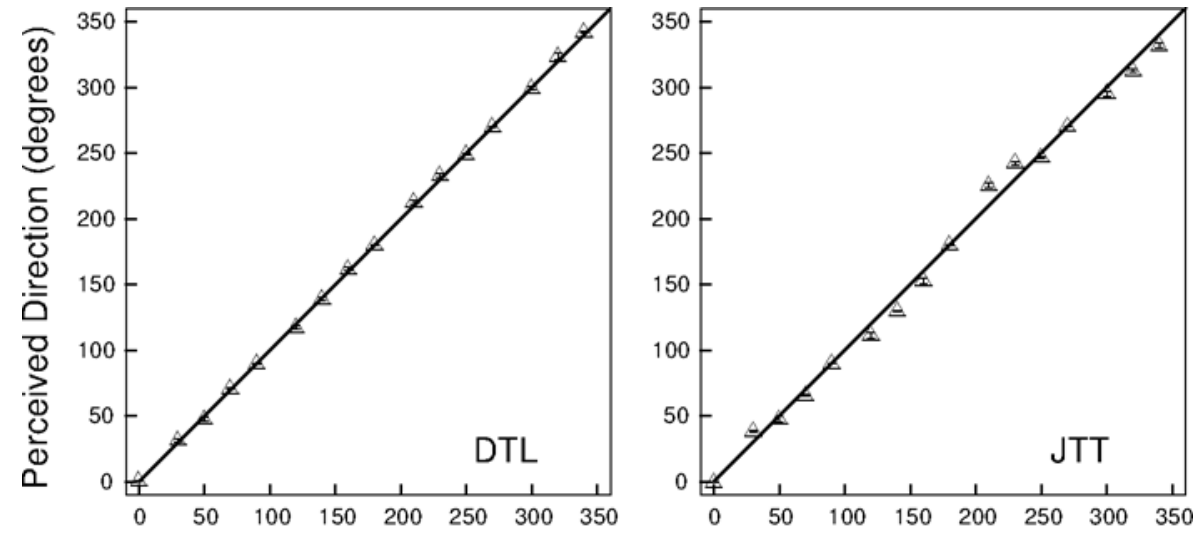

Direction of Motion (degrees)

Figure 4. Results of the control condition in Experiment 1. Average perceived directions of motion for a moving unfiltered texture. Error bars, \pm 1 SEM. Consult the text for further details.

with $1.6,1.07,0.53$, or $0.27 \mathrm{deg} / \mathrm{sec}$ oblique components. These combinations yielded speed ratios of $1: 1,3: 2,3: 1$, and 6:1, as is indicated in Figure 5.

The data are presented in a format that is used throughout this report. In this format, data obtained for each of the four cardinal directions have been pooled; each data point plotted in Figures 5 and 6 represents the average of 40 settings (4 cardinal directions per oblique direction $\times 10$ settings per direction). The cardinal directions have all been assigned a value of $0^{\circ}$, and the data have been plotted relative to that direction. The abscissa represents the relative direction of the oblique stimulus. The ordinate represents the relative perceived direction of motion of either the cardinal or the oblique component. Positive and negative values on both axes indicate directions counterclockwise and clockwise, respectively, from the direction of motion of the cardinal component. Squares represent the average settings of the perceived direction of the cardinal component when it is paired with each of the four oblique component values indicated on the ab-
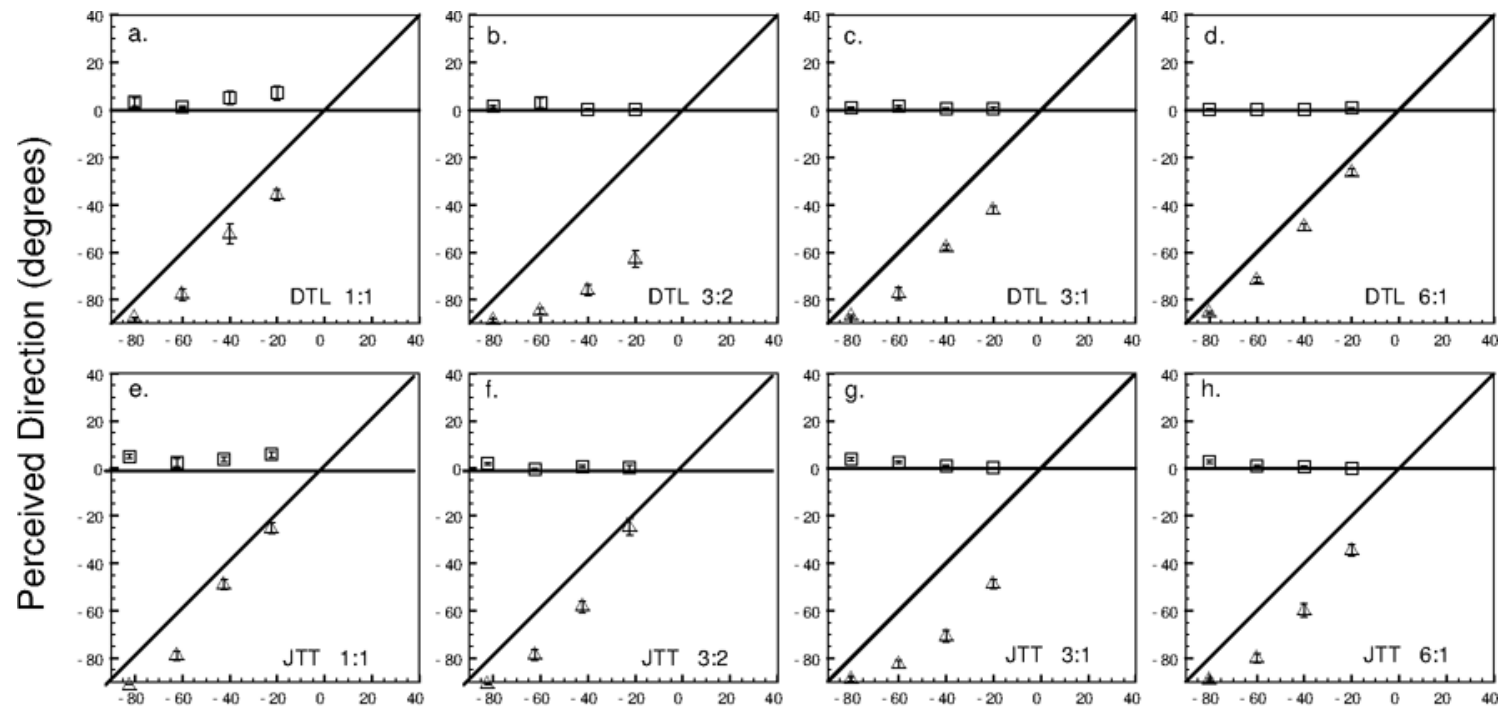

Relative Direction of Oblique Component (degrees)

Figure 5. Results of Experiment 1. Top row of graphs, subject D.T.L.; bottom row, subject J.T.T. Stimuli consisted of superposed unfiltered dynamic Julesz textures. Cardinal component speed was $1.6 \mathrm{deg} / \mathrm{sec}$. Each column plots the results for a different oblique component speed. (a and e) Oblique component speed was $1.6 \mathrm{deg} / \mathrm{sec}(\mathrm{speed}$ ratio $=1: 1)$. (b and f $) 1.07 \mathrm{deg} / \mathrm{sec}$ (3:2). (c and g) $0.53 \mathrm{deg} / \mathrm{sec}(3: 1)$. (d and h) $0.27 \mathrm{deg} / \mathrm{sec}(6: 1)$. Squares and triangles plot mean perceived directions of motion of the cardinal and oblique components, respectively. Error bars indicate $\pm 1 S E M$. See the text for description of the axis labels. 

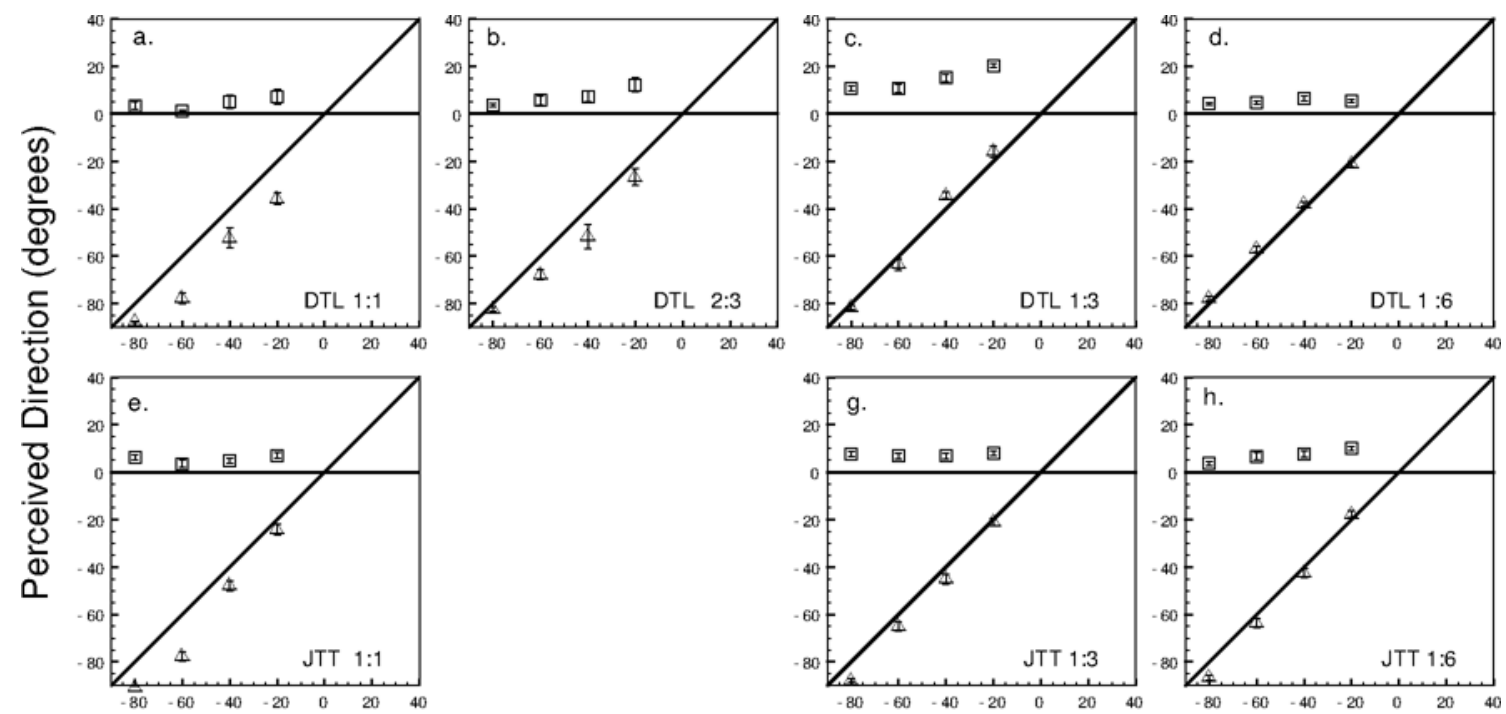

Relative Direction of Oblique Component (degrees)

Figure 6. Results of Experiment 1. Same as Figure 5, except that oblique component speed was held constant (1.6 deg/sec) and cardinal component speed was varied. (a and e) Cardinal component speed was $1.6 \mathrm{deg} / \mathrm{sec}(\mathrm{speed}$ ratio $=1: 1$ ), data replotted from Figure 5. (b) $1.07 \mathrm{deg} / \mathrm{sec}(2: 3)$. (c and g) $0.53 \mathrm{deg} / \mathrm{sec}(1: 3)$. (d and h) $0.27 \mathrm{deg} / \mathrm{sec}(1: 6)$.

scissa; triangles represent average settings of the corresponding oblique components. The horizontal and diagonal reference lines in each panel indicate the loci of true settings of cardinal and oblique directions, respectively. That is, in the absence of any interactions between components, the subjects' settings of cardinal direction (squares) should all fall on the horizontal line and their settings of oblique direction (triangles) should fall on the diagonal line. Settings of cardinal direction that fall above the horizontal reference line and settings of oblique direction that fall below the diagonal reference line indicate direction repulsion.

Both of the observers' results shown in Figures 5 and 6 exhibit three important features, which are summarized in Figure 7. First, direction repulsion is clearly evident at each of the component angular separations and

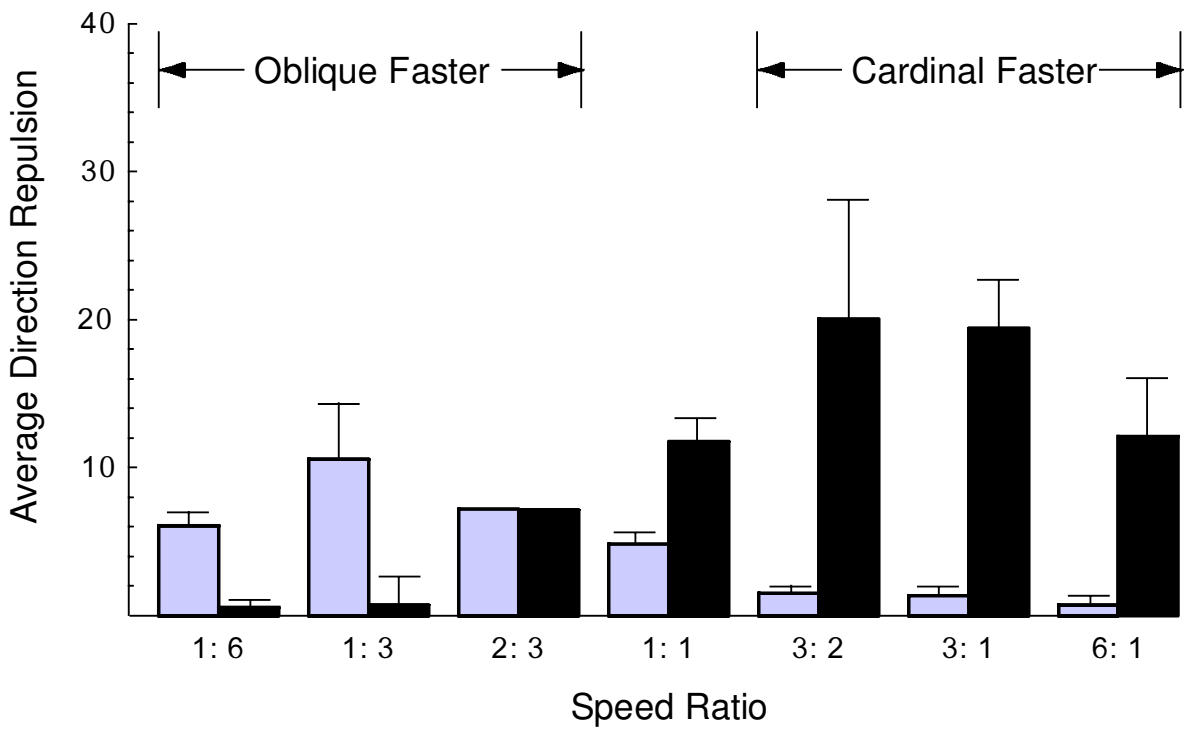

Figure 7. Summary of Experiment 1. Average direction repulsion is plotted in degrees as a function of the ratio of cardinal to oblique component speeds. Gray bars, settings of cardinal direction; black bars, settings of oblique direction. Each bar represents the average differences in degrees between the actual and the perceived directions of motion, pooled across angular separation and subject. Error bars, $1 S E M$. The results shown for speed ratio 2:3 are for one subject only (see Figure 6). 
speed combinations examined. Direction repulsion, on average, tends to vary inversely with the angular separation of the components over the range of separations investigated $\left(20^{\circ}-80^{\circ}\right)$. Direction repulsion for stimuli in which the cardinal and oblique components moved at the same speed is consistent with previous studies by Marshak and Sekuler (1979) and Hiris and Blake (1996), in which sparse random-dots patterns were used. This finding is not at all surprising, given that both kinds of patterns, random checks and sparse random dots, have similar spatial Fourier power spectra.

A second feature of the data is the clear dependence of direction repulsion on relative speed: The slower component generally exhibits the greater magnitude of direction repulsion. However, the data also suggest that the perception of cardinal and oblique directions of motion is not equally affected by differences in component speed: Direction repulsion is generally greater for the oblique component than for the cardinal component when data across reciprocal speed ratios are compared. For example, compare the results shown in Figure 7 for speed ratios of 3:1 (cardinal faster) and 1:3 (oblique faster). For either speed ratio, direction repulsion was greater in the slower moving component. Comparison across these conditions, however, shows that perception of the oblique component direction was more affected by the speed difference than was that of the cardinal component. Hiris and Blake (1996) have reported a similar motion anisotropy for superposed random-dot stimuli, although they examined only a single speed ratio of 1:1 in their study.

A third feature of the data is that they suggest that direction repulsion is mediated by interactions that exhibit some degree of speed tuning. This aspect of direction repulsion in random-check patterns is most clearly seen in Figure 7: Direction repulsion in the cardinal component appears to peak at a speed ratio of about 1:3, whereas repulsion in the oblique component appears to peak at a speed ratio in the range 3:2-3:1. Kim and Wilson (1996), using plaids and multiaperture bar patterns, also found that direction repulsion is greater in the slower moving component, although speed tuning was not observed in their study.

\section{Experiment 2}

In Experiment 1, the textures were unfiltered Julesz patterns, and when they were presented in superposition under the conditions described above, they always appeared to slide transparently past one another. In Experiment 2 , which involved ring-filtered textures, this was not always the case. Under some conditions, the two superposed patterns appeared to cohere into a single pattern moving in a direction intermediate between cardinal and oblique directions. The fraction of trials on which two patterns were seen, as opposed to a single pattern, depended on the spatial frequency composition of the patterns. The fraction of trials in Experiment 2 on which the subjects saw two patterns is shown in Figure 8. Each graph in the figure was generated by tallying the number of trials on which the subjects set two distinct directions of motion for the cardinal and oblique components generated. Results are shown for each of the three textures employed in Experiment 2, collapsed across angular separation and speed ratio. The figure shows that two patterns were almost always seen when the stimuli consisted of 2or 4-cpd ring-filtered textures. On the other hand, the subjects clearly had greater difficulty in setting two directions of motion when the stimuli consisted of 1-cpd textures.

Figures 9-11 show the perceived directions of motion of the cardinal and oblique textures on those trials in which the subjects made two distinct settings. The results are averages of the subjects' settings, collapsed across the four cardinal directions tested, as was described earlier. Each of these figures shows data for a different center frequency of filtering. In each figure, the left column shows data obtained when both cardinal and oblique components moved at $1.6 \mathrm{deg} / \mathrm{sec}(\mathrm{FF})$. The other two columns of graphs show results for stimuli that had cardinal or oblique components moving at different speeds: 1.6 versus $0.27 \mathrm{deg} / \mathrm{sec}$. The middle column shows data for oblique-faster conditions (SF), whereas the right column of graphs shows data for cardinal-faster conditions (FS).

If we consider the 2- and 4-cpd data first (Figures 10 and 11), they exhibit some of the features of motion per-
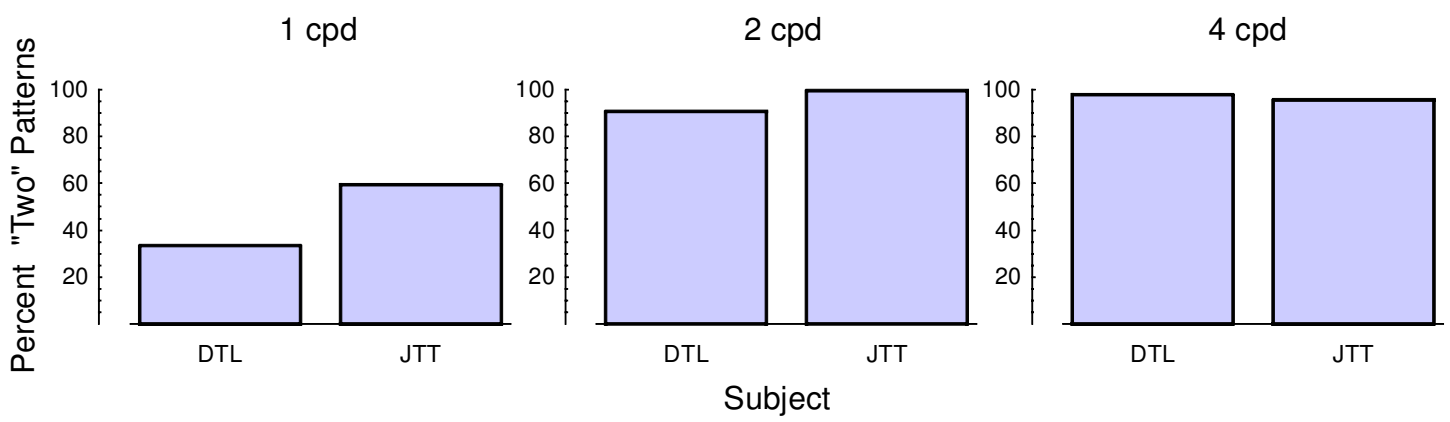

Figure 8. Percentages of trials in Experiment 2 on which the stimulus was perceived as two moving patterns, based on subjects' settings of cardinal and oblique directions in a given trial. If the two settings differed by more than $5^{\circ}$, the trial was scored as two; otherwise, the trial was scored as one. 

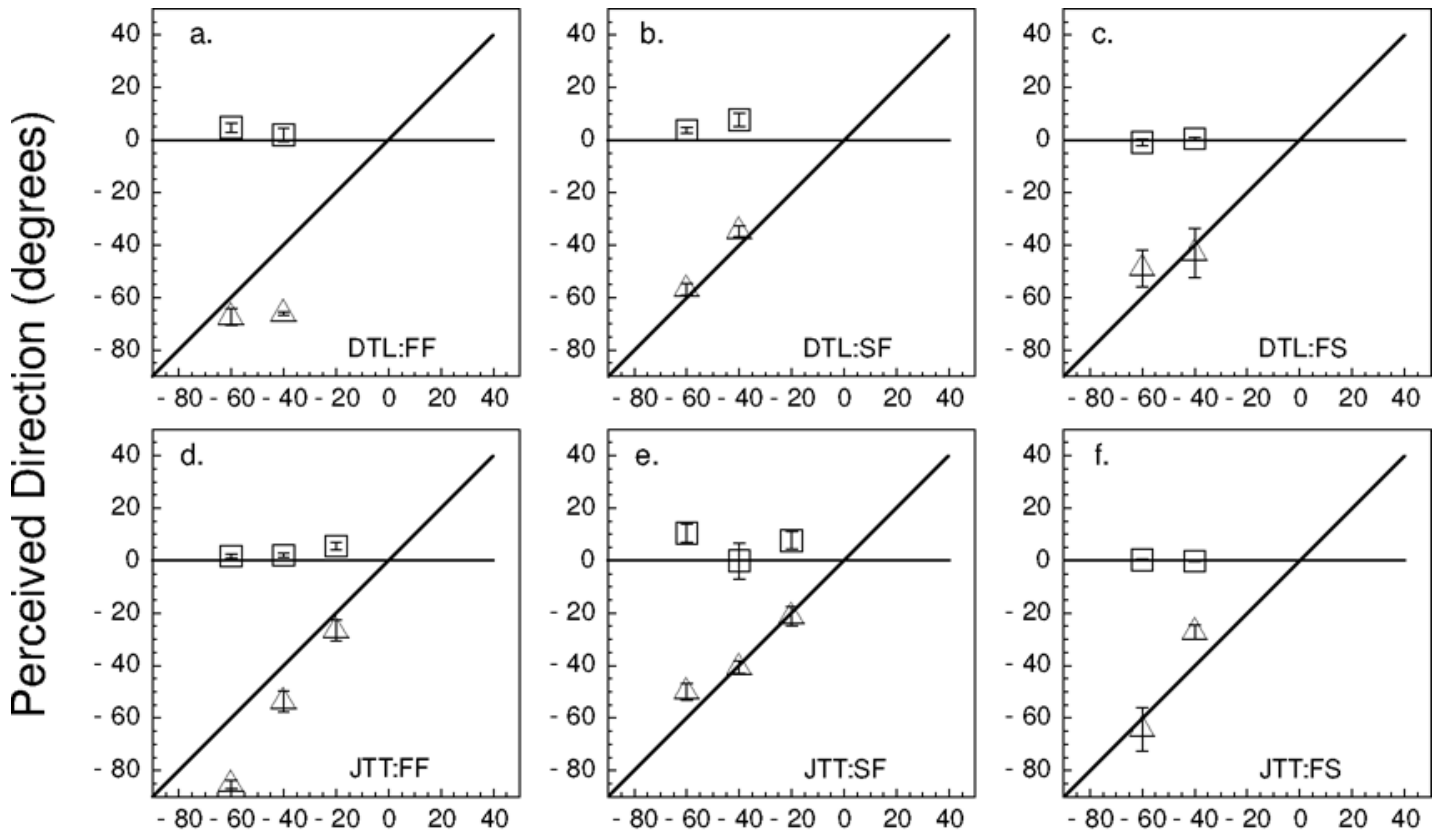

\section{Relative Direction of Oblique Component (degrees)}

Figure 9. Results of Experiment 2. Average perceived directions of cardinal (squares) and oblique (triangles) components as functions of oblique component direction. Both cardinal and oblique components are 1-cpd ring-filtered Julesz textures. (a-c) Data for subject D.T.L. (d-f) Data for subject J.T.T. FF, cardinal and oblique textures move at $1.6 \mathrm{deg} / \mathrm{sec} ; \mathrm{SF}$, cardinal/oblique speeds are $0.27 / 1.6 \mathrm{deg} / \mathrm{sec} ; \mathrm{FS}$, cardinal/oblique speeds are $1.6 / 0.27 \mathrm{deg} / \mathrm{sec}$. Error bars, $\pm 1 S E M$. Consult the text for further details.
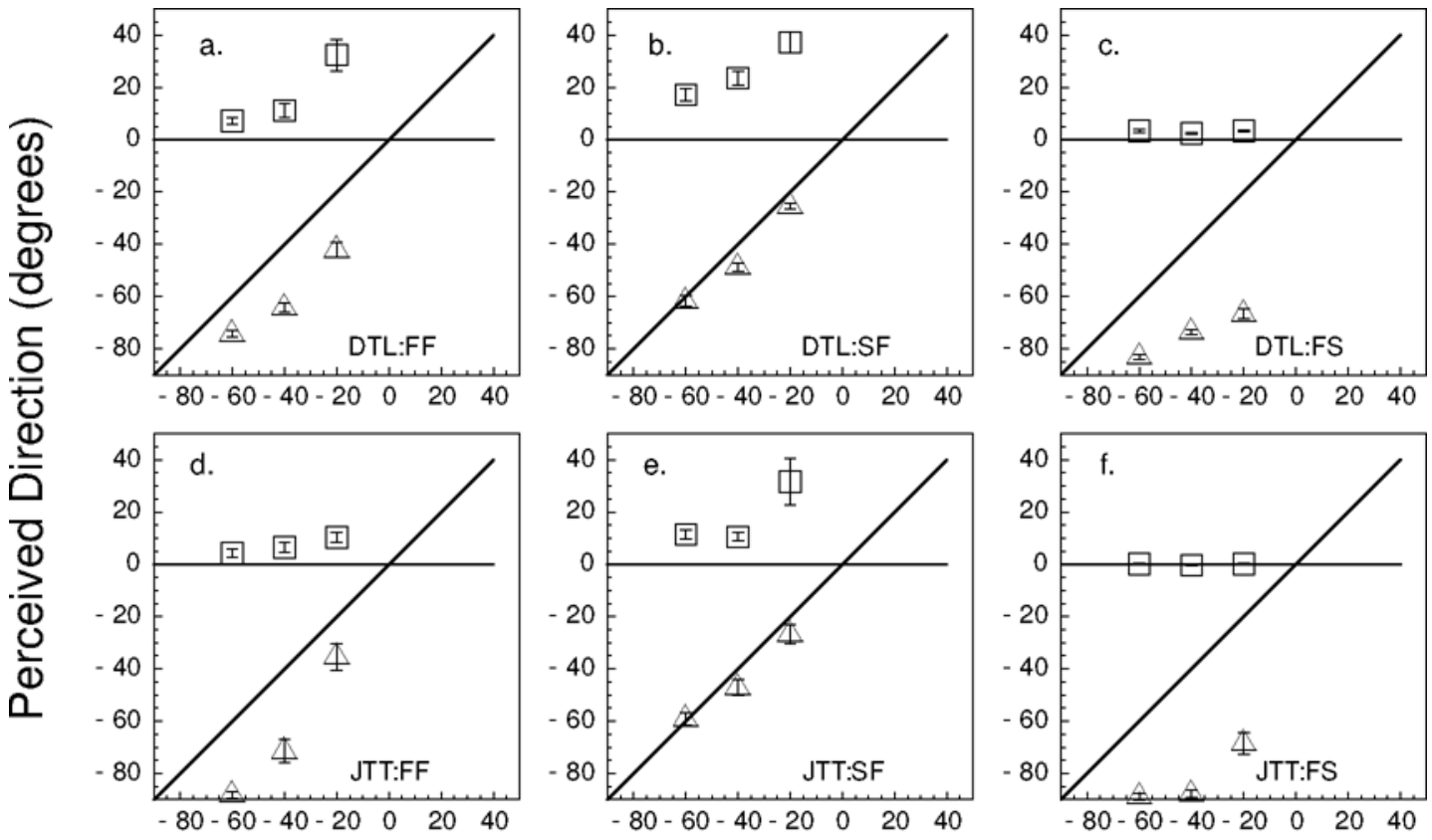

Relative Direction of Oblique Component (degrees)

Figure 10. Results of Experiment 2. Cardinal and oblique components are 2.0-cpd ring-filtered Julesz textures. The format of this figure is the same as that for Figure 9. Consult the text for further details. 

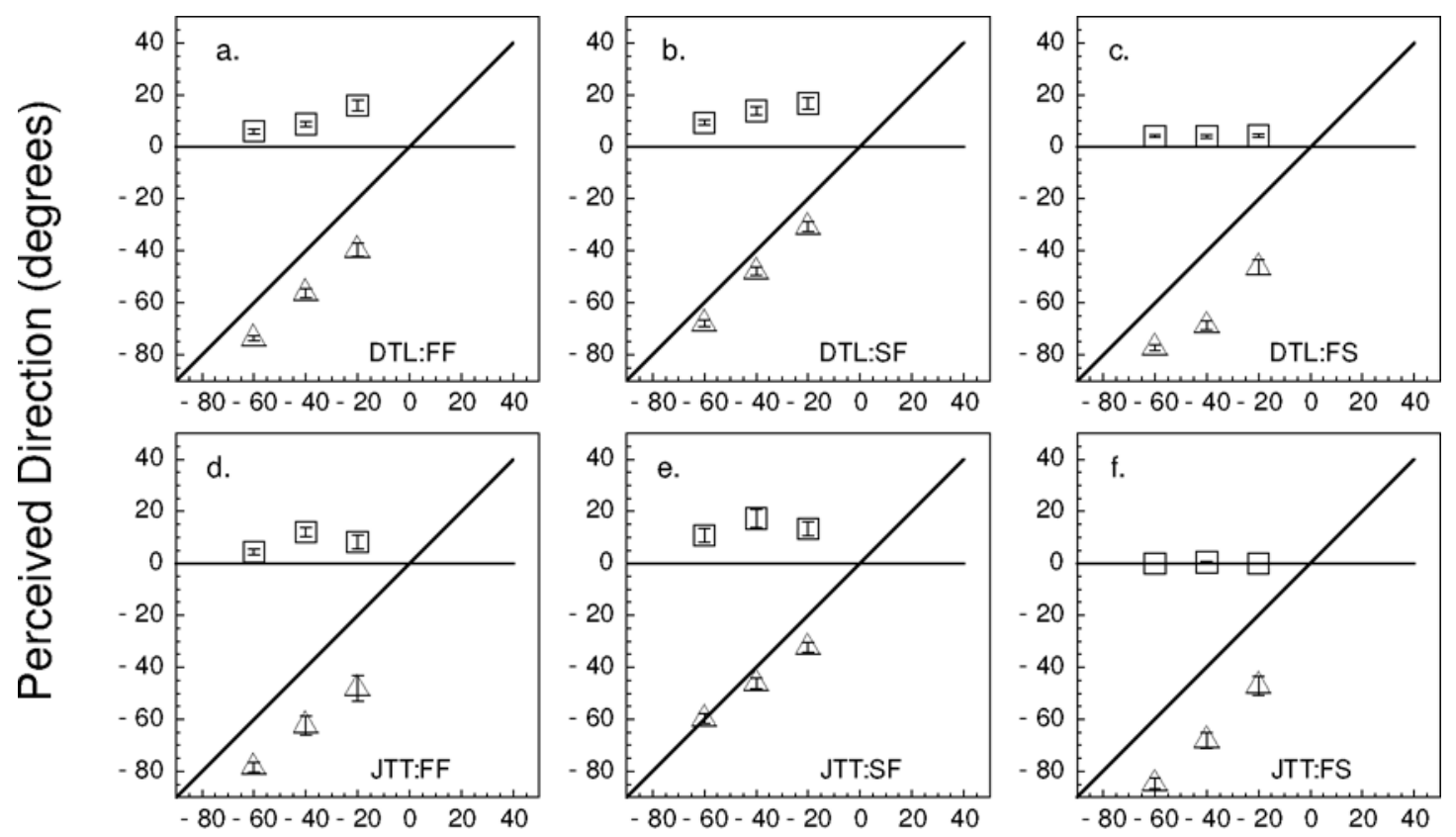

\section{Relative Direction of Oblique Component (degrees)}

Figure 11. Results of Experiment 2. Cardinal and oblique components are 4.0-cpd ring-filtered Julesz textures. The format of this figure is the same as that for Figure 9. Consult the text for further details.

ception observed with unfiltered textures. First, they show that direction repulsion occurred in either or both components, regardless of the angular separation or speed combinations tested. Thus, interactions leading to motion repulsion span at least the same sixfold range of speeds observed for unfiltered textures in Experiment 1. Second, a comparison of the results shown in the middle and right-hand columns of the graphs in Figures 10 and 11 shows that direction repulsion in 2- and 4-cpd textures is greater in the slower moving of the cardinal and oblique components. This can be seen more clearly in the summary plots shown in Figures $12 \mathrm{~b}$ and $12 \mathrm{c}$. Third, the data plotted in Figures 10 and 11 and summarized in Figures $12 \mathrm{~b}$ and $12 \mathrm{c}$ also show the same cardinal/oblique direction repulsion anisotropy seen with unfiltered textures.
Whereas the results for direction repulsion obtained with 2- and 4-cpd textures are qualitatively similar to those obtained with unfiltered Julesz textures, the results obtained for the 1 -cpd conditions are somewhat different. As the results shown in Figure 8 indicate, the 1-cpd cardinal and oblique components have a tendency to cohere. Thus, settings of separate directions of motion for these stimuli were generally much more difficult to make than those for the 2- or 4-cpd textures. Settings for the smallest angular separations of the components $\left(20^{\circ}\right)$ were especially difficult for subject D.T.L. (see Figures $9 \mathrm{a}, 9 \mathrm{~b}$, and 9c). Examination of the summary of results for 1-cpd textures (Figure 12a) shows that, overall, direction repulsion was greatly diminished at this coarser spatial scale, except for the conditions in which both tex-

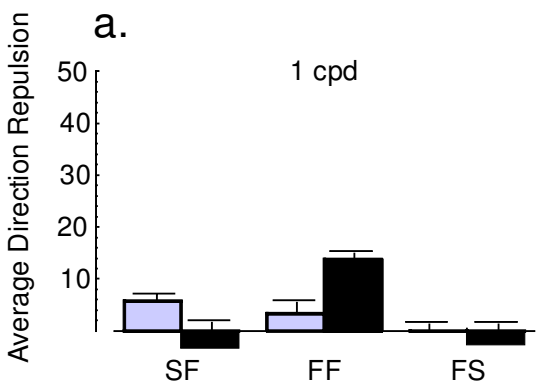

b.

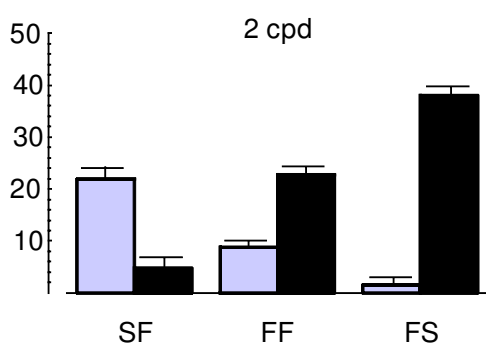

C.

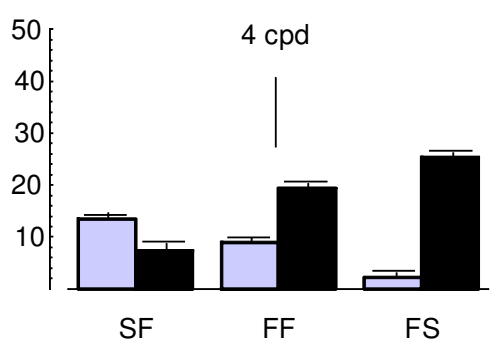

Figure 12. Summary of Experiment 2. (a-c) Results for 1-, 2-, 4-cpd ring-filtered textures, respectively. Bars in each graph correspond to average repulsion in degrees for cardinal (gray bars) or oblique (black bars) components. Average repulsion was computed across three angular separations $\left(2^{\circ}, 40^{\circ}\right.$, and $\left.60^{\circ}\right)$ and 2 subjects. Error bars represent $1 S E M$. Abscissa labels represent relative speeds (slow vs. fast) of cardinal/oblique components. SF, 0.27/1.6 deg/sec; FF, 1.6/1.6 deg/sec; FS, 1.6/ 0.27 deg/sec. 


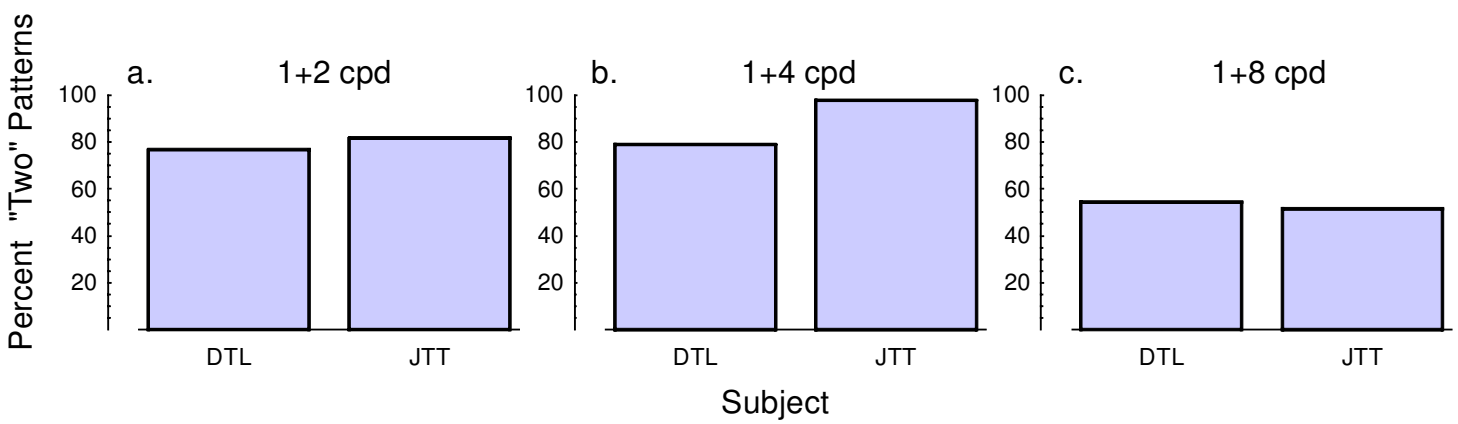

Figure 13. Percentages of trials in Experiment 3 on which the stimulus was perceived as two moving patterns. See Figure 8 and the text for further details.

tures moved at the same $1.6 \mathrm{deg} / \mathrm{sec}$ speed. Note that these conditions also reveal the same motion anisotropy observed for the other textures.

\section{Experiment 3}

Figures 13-17 show the effects of pairing a 1-cpd ringfiltered cardinal component with a 2-, 4-, or 8-cpd oblique component. When the patterns differed in spatial scale by a factor of 2 (panel a, 1 vs. 2 cpd) or a factor of 4 (panel b, 1 vs. 4 cpd), two patterns were seen on about $80 \%$ or more of the trials (Figure 13). When the component textures differed eightfold in center spatial frequency, they had a tendency to cohere approximately $50 \%$ of the time (panel c, 1 vs. $8 \mathrm{cpd}$ ). The perceived direction of global pattern motion in these cases appears to be determined either by the direction of the faster component or by the direction of the cardinal component.

The average settings of perceived direction on those trials in which the subjects could make distinct settings of cardinal and oblique component directions are shown in Figures 14-16. The data obtained with a 2- or 4-cpd oblique component (Figures 14 and 15) show clearly that interactions leading to direction repulsion in transparency are sustained across differences in spatial scale of 1 and 2 octaves, as well as across sixfold speed differences between cardinal and oblique conditions. Asymmetries in direction repulsion owing to differences in cardinal and oblique component speed are also apparent. These ef-
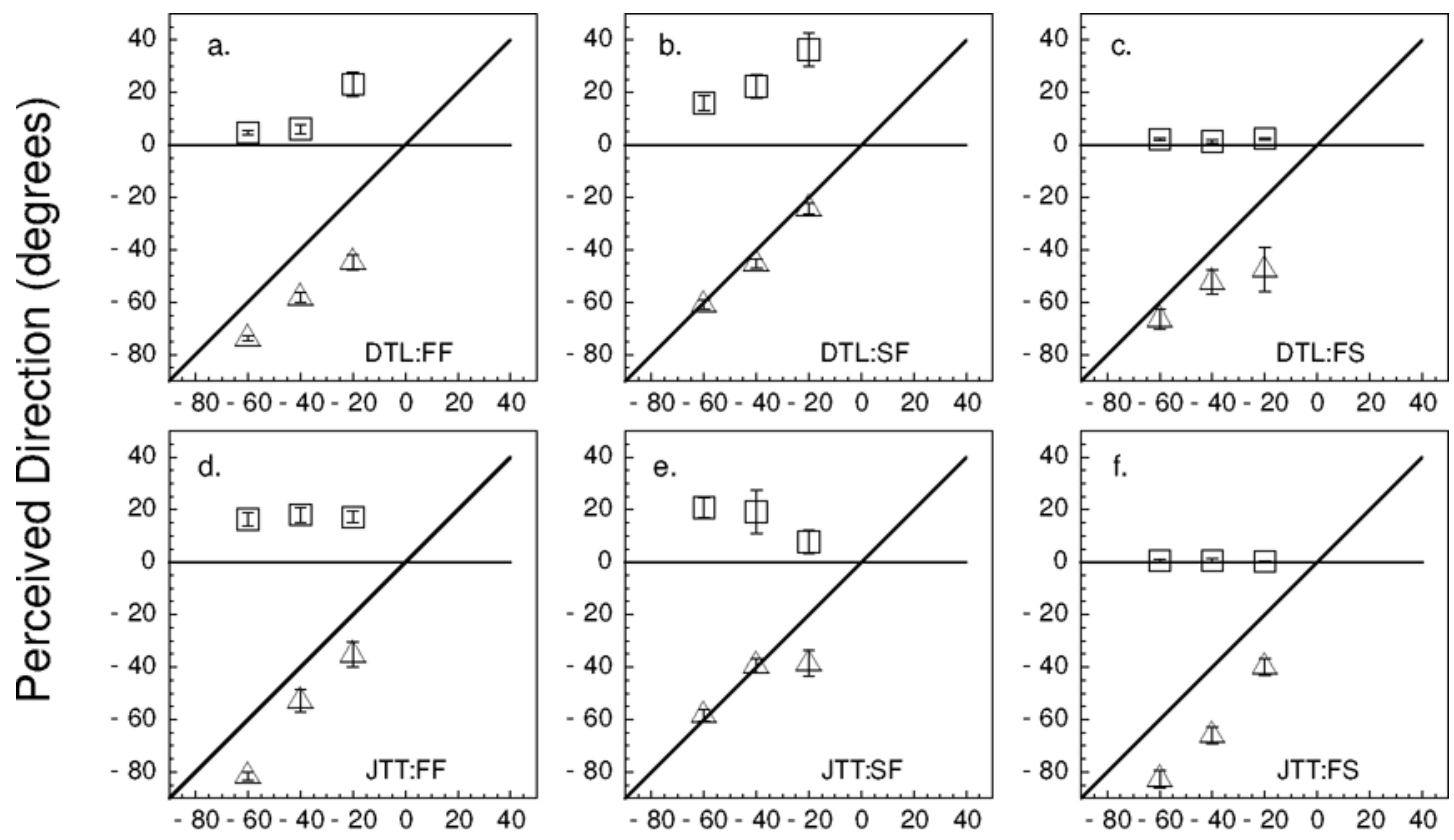

Relative Direction of Oblique Component (degrees)

Figure 14. Results of Experiment 3. Average perceived directions of cardinal (squares) and oblique (triangles) components as functions of the oblique component direction for ring-filtered Julesz textures. Cardinal textu re, 1 cpd; oblique texture, $2 \mathrm{cpd}$. The format of this figure is the same as that for Figure 9 . Consult the text for further details. 

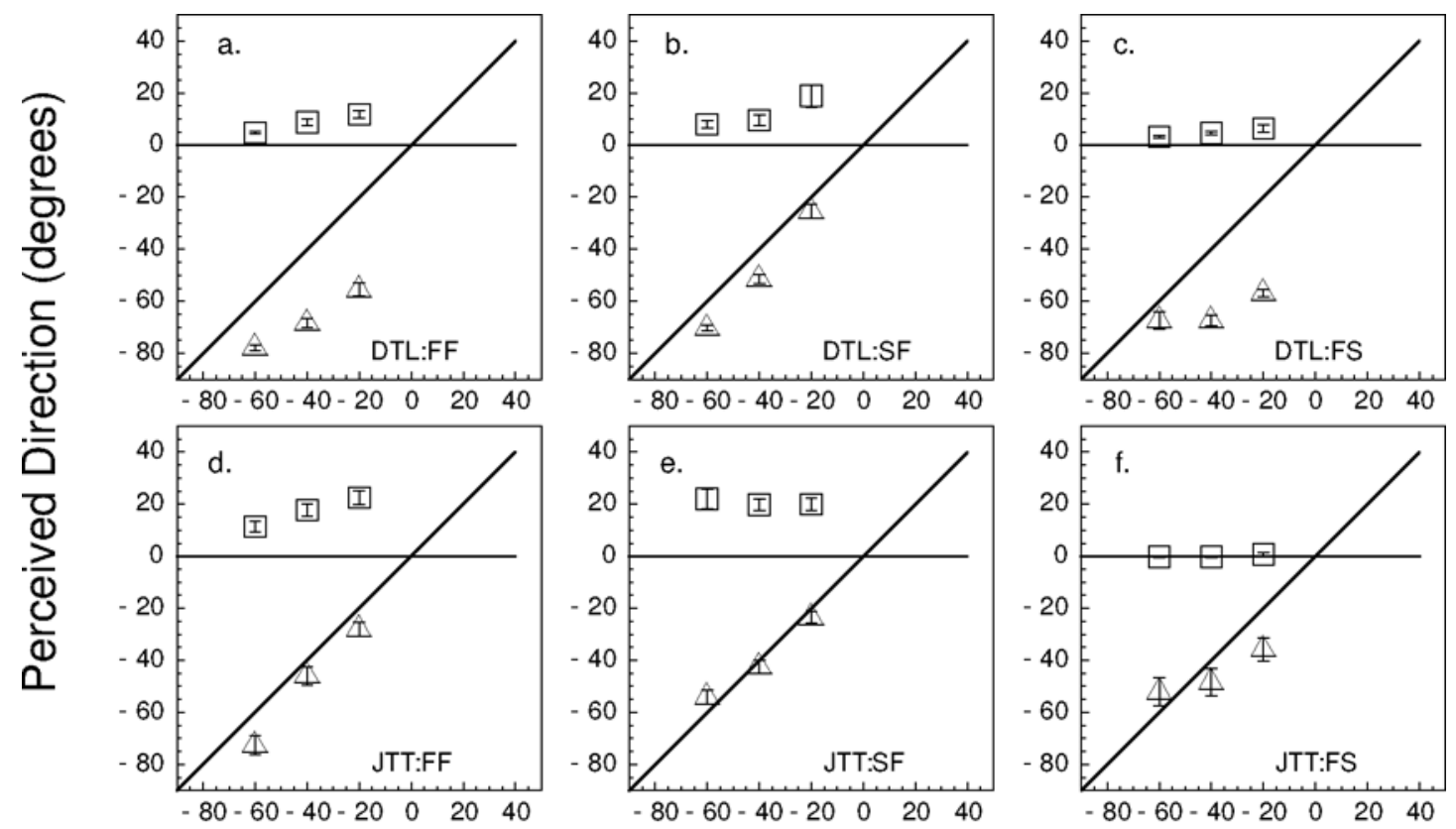

Relative Direction of Oblique Component (degrees)

Figure 15. Results of Experiment 3. Average perceived directions of cardinal (squares) and oblique (triangles) components as functions of the oblique component direction for ring-filtered Julesz textures. Cardinal textu re, 1 cpd; oblique texture, $4 \mathrm{cpd}$. The format of this figure is the same as that for Figure 9. Consult the text for further details.
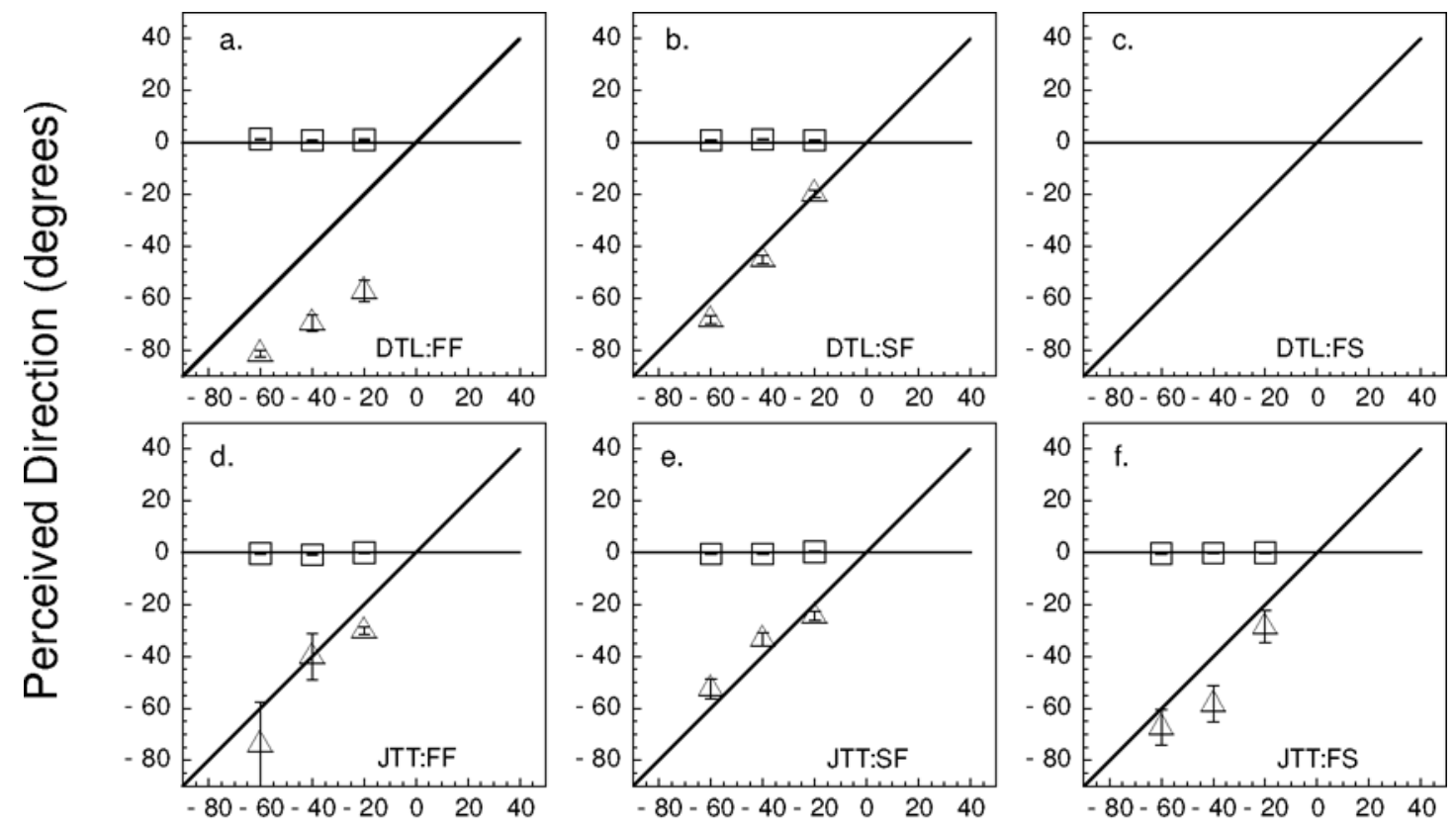

Relative Direction of Oblique Component (degrees)

Figure 16. Results of Experiment 3. Average perceived directions of cardinal (squares) and oblique (triangles) components as functions of the oblique component direction for ring-filtered Julesz textures. Cardinal textu re, 1 cpd; oblique texture, $8 \mathrm{cpd}$. The format of this figure is the same as that for Figure 9 . Consult the text for further details. 


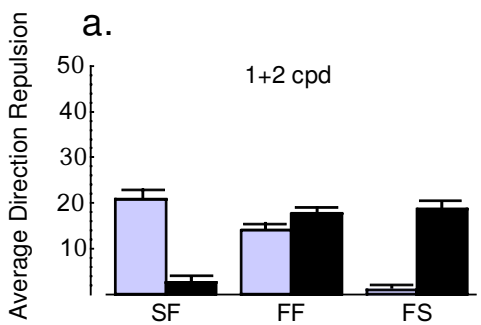

b.

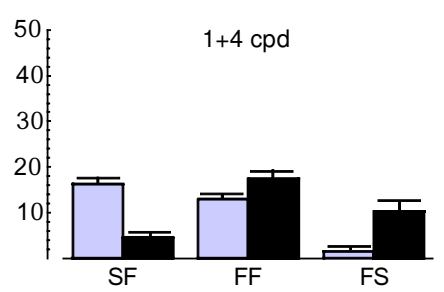

C.

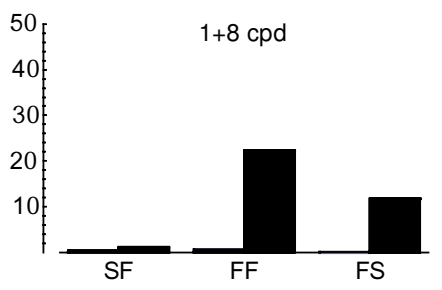

Figure 17. Summary of Experiment 3. Average repulsion data in degrees for three speed combinations. In all cases, the cardinal component is a 1-cpd ring-filtered texture. (a-c) Results for 2-, 4-, and 8-cpd ring-filtered oblique components, respectively. Graphic conventions as in Figure 12.

fects of speed ratio on direction repulsion are more clearly seen in the summary Figures $17 \mathrm{a}$ and $17 \mathrm{~b}$.

The data obtained when the patterns differed in spatial scale by 3 octaves (Figure 16, Figure 17c) are more difficult to interpret. Although the data indicate a dependence of direction repulsion on the relative speed of the components, the data also indicate that direction repulsion occurred only for the oblique component of these stimuli. In any event, the results of Experiment 3 clearly show interactions that span at least a fourfold range of spatial frequencies and a sixfold range of speed differences.

\section{DISCUSSION}

The present series of three experiments was designed to investigate the spatiotemporal range of interactions that lead to direction repulsion. For reasons that were developed in the introduction, these interactions are thought to be important to the perceptual segregation of superposed dynamic textures into their moving component parts. In Experiment 1, the stimuli were unfiltered dynamic Julesz textures; the following results were obtained. (1) Direction repulsion occurred across a sixfold range of cardinal and oblique component speeds. Direction repulsion also occurred across the entire range of angular separations of components investigated. (2) In general, an increase in the speed of one component tended to produce an increased direction repulsion in the other, although there was also some degree of speed tuning. (3) Motion anisotropy also occurred in Experiment 1: Direction repulsion was generally weaker in the cardinal directions, and the effects of relative speed were less pronounced, than in the oblique directions.

In Experiment 2, the stimuli consisted of ring-filtered cardinal and oblique textures with the same center frequencies: 1, 2, or $4 \mathrm{cpd}$. The results at 2 and $4 \mathrm{cpd}$ replicated all of the basic findings of Experiment 1 . On the other hand, both the occurrence of transparency and the magnitude of direction repulsion were greatly reduced for the 1-cpd stimuli, suggesting a lower limit on the spatiotemporal frequencies that support motion transparency.

Finally, in Experiment 3, a 1-cpd texture was paired with a 2-, 4-, or 8-cpd texture. The results show that interactions that underlie direction repulsion are sustained across at least a fourfold range of spatial frequencies, as well as a sixfold range of speed ratios. At the extreme of this range, when 1- and 8-cpd textures were paired, transparency was seen less often, and on those trials in which transparency was seen, there was a smaller effect of speed difference on direction repulsion. This tendency suggests that there is also an upper limit on the spatiotemporal extent of the underlying interactions.

Thus, repulsion in ring-filtered textures spans a wide spatiotemporal frequency range. This result is consistent with the results of numerous previous theoretical and empirical studies of the interactions among motion signals across spatial scale (e.g., Ido, Ohtami, \& Ejima, 1997; Kim \& Wilson, 1993, 1996; Yang \& Blake, 1994) and speed (e.g., Kim \& Wilson, 1996; Watamaniuk, Sekuler, \& Williams, 1989; Williams \& Phillips, 1987). Most investigators have been interested primarily in understanding how the visual system achieves a single, integrated, coherent motion percept from the responses of motion sensors tuned to different spatial frequencies and/or different speeds. Less attention has been paid to the question of how interactions across spatial frequencies and/ or speeds contribute to direction repulsion and the computation of motion transparency.

To my knowledge, Kim and Wilson (1996) published the only previous study to address spatiotemporal frequency effects in direction repulsion. There is good agreement between Kim and Wilson's (1996) results and the results of Experiment 1. Both studies find similar effects of relative component speed on direction repulsion, although Kim and Wilson (1996) found that direction repulsion in the slower component varied approximately linearly with speed difference between components, whereas the results of the present study indicate that the effects of speed difference may be nonmonotonic.

Kim and Wilson (1996) also investigated the effects of component spatial frequency difference on direction repulsion. They found that direction repulsion occurred only when the two stimulus components had the same spatial frequency. This result is somewhat different from those of the present study, which show direction repulsion across at least a fourfold difference in component spatial frequency. However, Kim and Wilson (1996) used stimuli consisting of moving one-dimensional (1-D) com- 
ponents - that is, bars and gratings. These stimuli almost always cohere, and when they do, they produce a single unified global motion percept. This tendency toward coherence may be disrupted somewhat if one manipulates the relative speeds, colors, contrasts, and spatial frequencies of the components (e.g., Adelson \& Movshon, 1982; Kooi, DeValois, Switkes, \& Grosof, 1992; Krauskopf \& Farell, 1990; Movshon, Adelson, Gizzi, \& Newsome, 1985; Stone, Watson, \& Mulligan, 1990). Coherence may also be disrupted when the stimuli are plaids made of rectangular-wave gratings that simulate the surface properties of overlapping, phenomenally transparent bars (Lindsey \& Todd, 1996; Stoner \& Albright, 1996; Stoner, Albright, \& Ramachandran, 1990; Trueswell \& Hayhoe, 1993). Motion adaptation may also augment the effects of phenomenal transparency on plaid coherence (Lindsey \& Todd, 1996). Nonetheless, these manipulations are generally ineffective in producing motion transparency unless the angular separation of the components is $90^{\circ}$ or more. On the other hand, superposed dynamic random textures almost always fail to cohere. Several studies (e.g., Hiris \& Blake, 1996; Marshak \& Sekuler, 1979; van Doorn $\&$ Koenderink, 1983), including the present one, suggest that angular separations of as little as $20^{\circ}-30^{\circ}$ are sufficient for the perception of motion transparency in these patterns. The results of the present study are particularly compelling because they are derived from a task in which subjects must represent perceptually two directions of motion simultaneously, rather than merely segregate one motion component from the background activity (see Braddick, 1997, for further discussion of this point).

The differences between plaids and dynamic Julesz patterns noted above may be due to interactions occurring among frequency components present in the Julesz patterns, which are 2-D stimuli, that are not present in the $1-D$ stripes that make up the plaid stimuli. For example, the 1-D components of plaid stimuli have all their spatial Fourier power concentrated along one of two orientations. On the other hand, 2-D textures, such as those used in the present series of experiments, have their spatial Fourier power distributed widely across orientation. The results of the present study are consistent with this view. What remains unclear is precisely how the additional spatial frequency information in 2-D random textures is utilized. The finding that motion transparency is observed in two 2-D textures whose directions of motion are separated by $20^{\circ}-30^{\circ}$ places an important constraint on models of motion perception that attempt to account for human motion transparency. Recent models that have attempted to do so (e.g., Simoncelli \& Heeger, 1998; Wilson \& Kim, 1994) incorporate low-level sensors that are rather broadly tuned to motion (orientation bandwidths of around $45^{\circ}$, spatiotemporal bandwidths of 1-2 octaves). An important property of these models is that they rely greatly on angular separation between the components of the stimulus to partition the signals that might be associated with each pattern in a transparent motion stimulus. That is, in order for these models to resolve the overall distributions of motion energy into two distinct sources of motion, there must be a bimodal pattern in the population responses of early visual mechanisms to the composite stimulus. Subsequent stages of processing that involve excitatory or inhibitory interactions among these mechanisms serve to successively sharpen the selectivity of the model for different directions and/or velocities of motion present in the composite stimulus. In these models, direction repulsion results from the inhibitory interactions occurring at these subsequent stages of processing.

These models appear to be well suited to the analysis of motion transparency in grating and bar pattern stimuli, since an angular selectivity of no more than $80^{\circ}-90^{\circ}$ is suggested by empirical investigations of motion transparency in these stimuli. On the other hand, each 2-D component in the stimuli used in the present study excites sensors tuned to a wide range of directions. When two such patterns of sensor responses overlap, the final distribution of responses will not be bimodal when the angular separation of the components is on the order of $20^{\circ}-30^{\circ}$. It does not seem possible that models of motion perception like those outlined above could resolve the component motions in these stimuli.

A way out of this dilemma that has been pursued recently is to assume that the visual system takes explicit advantage of the statistical properties of dynamic random textures (e.g., Nowlan \& Sejnowski, 1995; Qian, Andersen, \& Adelson, 1994b). These models take advantage of the fact that there will be random spatial variations in the local Fourier power spectrum of each component in a compound dynamic random-texture stimulus. Local "islands "of high activity that are due to one or the other component are weighted more heavily in the estimation of global pattern motion than are responses from regions in which both patterns contribute significantly to the estimation of motion energy. In Qian et al.'s (1994b) model, inhibitory interactions among local motion sensors tuned to different directions of motion are used to implement the weighting function. It is possible, however, that these processes may not be involved in direction repulsion. Psychophysical studies by Qian and colleagues (Qian et al., 1994a), for example, have shown that the proposed inhibitory processes do not operate when the two moving patterns are separated in depth. On the other hand, the magnitude of direction repulsion is unaffected by this stimulus manipulation (Hiris \& Blake, 1996). Clearly, more work on the relationship between motion energy segregation and direction repulsion is needed.

Finally, we turn to the finding that direction repulsion is anisotropic - that is, pattern repulsion in a transparent motion stimulus is generally less for the cardinal than for the oblique component. The present study replicates and extends a recent study by Hiris and Blake (1996) in which the authors used sparse random-dot arrays. In their 
study, direction repulsion in the oblique component reached a maximum of about $10^{\circ}$ for angular separations of $20^{\circ}-40^{\circ}$ from the cardinal component, whereas repulsion in the four cardinal directions was never more than about $2^{\circ}$. The authors only studied the condition where the two patterns moved at the same speed. The results of the present study show that the direction anisotropy in motion repulsion occurs across a wide range of cardinal/oblique speed differences, provided that both components have the same or similar spatial frequency composition.

The basis for this direction repulsion anisotropy remains uncertain. Hiris and Blake (1996) postulated that it might reflect a preponderance of neurons in the human visual system tuned to motion in the cardinal directions. If so, one might expect motion sensitivity in the cardinal directions to be greater than that in other directions. This does not appear to be the case (see Gros, Blake, \& Hiris, 1998). One possible alternative explanation for repulsion anisotropy may be that it reflects direction-specific differences in the relative weights of excitatory and inhibitory interactions in networks involved in the processing of transparent motion. Another possibility is that cardinal directions are perceptually privileged and that "higher order" processes, such a perceptual anchoring, partially override lower level, motion-based processes that produce direction repulsion. Further work is needed to gain a better understanding of the causes of direction repulsion anisotropy.

\section{REFERENCES}

Adelson, E. H., \& Movshon, J. A. (1982). Phenomenal coherence of moving visual patterns. Nature, 300, 523-525.

BRADDICK, O. J. (1997). Local and global representations of velocity; transparency, opponency, and global direction perception. Perception, 26, 995-1010.

Chang, J. J., \& Julesz, B. (1983). Displacement limits for spatial frequency filtered random-dot cinematograms in apparent motion. $\mathrm{Vi}$ sion Research, 23, 1379-1385.

Cleary, R., \& Braddick, O. J. (1990). Direction discrimination for band-pass filtered random dot kinematograms. Vision Research, 30, 303-316.

GIESE, M. A. (1999). A dynamical model for the perceptual organization of apparent motion. Boston: Kluwer.

Gros, B. L., BlaKe, R., \& Hiris, E. (1998). Anisotropies in visual motion perception: A fresh look. Journal of the Optical Society of America A, 15, 2003-2011.

Hiris, E., \& Blake, R. (1996). Direction repulsion in motion transparency. Visual Neuroscience, 13, 187-197.

Ido, K., Ohtami, Y., \& Euma, Y. (1997). Dependencies of motion assimilation and motion contrast on spatial properties of stimuli: Spatialfrequency nonselective and selective interactions between local motion detectors. Vision Research, 37, 1565-1574.

KIM, J., \& WiLson, H. R. (1993). Dependence of plaid motion coherence on component grating directions. Vision Research, 33, 2479-2489.

Kim, J., \& Wilson, H. R. (1996). Direction repulsion between components in motion transparency. Vision Research, 36, 1177-1187.

Kooi, F., DeValois, K. K., Switkes, E. \& Grosof, X. (1992). Higher order factors influencing the perception of sliding and coherence of a plaid. Perception, 21, 583-598.
KrausKopf, J., \& FAREll, B. (1990). Influence of color on the perception of coherent motion. Nature, 348, 328-331.

Lindsey, D. T., \& TodD, J. T. (1996). On the relative contributions of motion energy and transparency to the perception of moving plaids. Vision Research, 36, 207-222.

LindSEY, D. T., \& TodD, J. T. (1998). Opponent motion interactions in the perception of transparent motion. Perception \& Psychophysics, 60, 558-574.

Marshak, W., \& Sekuler, R. (1979). Mutual repulsion between moving visual targets. Science, 205, 1399-1401.

Mather, G., \& Moulden, B. (1980). A simultaneous shift in apparent direction: Further evidence for a "distribution-shift" model of direction coding. Quarterly Journal of Experimental Psychology, 32, 325-333.

MATHER, G., \& Moulden, B. (1983). Thresholds for movement direction: Two directions are less detectable than one. Quarterly Journal of Experimental Psychology, 35A, 513-518.

Mingolla, E., Todd, J. T., \& Norman, J. F. (1983). The perception of globally coherent motion. Vision Research, 32, 1015-1031.

Movshon, J. A., Adelson, E. H., Gizzi, M. S., \& Newsome, W. T. (1985). The analysis of moving visual patterns. In C. Chagas, R. Gattass, \& C. Gross (Eds.), Pattern recognition mechanisms (Vol. 54, pp. 117-151). Rome: Vatican Press.

Nowlan, S. J., \& SeJnowsKi, T. J. (1995). Filter selection model for motion segmentation and velocity integration. Journal of the Optical Society of America A, 11, 3177-3200.

Press, W. H., Teukolsky, S. A., Vetterling, W. T., \& Flannery, B. P. (1993). Numerical recipes in C: The art of scientific computing (2nd ed.). New York: Cambridge University Press.

QIAN, N., \& ANDERSEN, R. A. (1994). Transparent motion perception as detection of unbalanced signals: II. Physiology. Journal of Neuroscience, 14, 7367-7380.

Qian, N., Andersen, R. A., \& Adelson, E. H. (1994a). Transparent motion perception as detection of unbalanced signals: I. Psychophysics. Journal of Neuroscience, 14, 7357-7366.

Qian, N., Andersen, R. A., \& Adelson, E. H. (1994b). Transparent motion perception as detection of unbalanced signals: III. Modeling. Journal of Neuroscience, 14, 7381-7392.

Simoncelli, E. P., \& HeEger, D. J. (1998). A model of neuronal responses in visual area MT. Vision Research, 38, 743-761.

SNOWDEN, R. J. (1989). Motions in orthogonal directions are mutually suppressive. Journal of the Optical Society of America A, 6, 1096-1101.

SNOWDEN, R. J. (1990). Suppressive interactions between moving patterns: Role of velocity. Perception \& Psychophysics, 47, 74-78.

Snowden, R. J., Treue, S., Erikson, R. G., \& Andersen, R. A. (1991). The response of area MT and V1 neurons to transparent motion. Journal of Neuroscience, 11, 2768-2785.

Stone, L. S., Watson, A. B., \& Mulligan, J. B. (1990). Effect of contrast on the perceived direction of a moving plaid. Vision Research, 30, 1049-1067.

Stoner, G. R., \& Albright, T. D. (1996). The interpretation of visual motion: Evidence for surface segmentation mechanisms. Vision Research, 36, 1291-1310.

Stoner, G. R., Albright, T. D., \& Ramachandran, V. S. (1990). Transparency and coherence in human motion perception. Nature, 344, 153-155.

Stromeyer, C. F., Kronauer, R. E., Madsen, J. C., \& Klein, S. A. (1984). Opponent-movement mechanisms in human vision. Journal of the Optical Society of America A, 1, 876-884.

Trueswell, J. C., \& Hayhoe, M. M. (1993). Surface segmentation mechanisms and motion perception. Vision Research, 33, 313-328.

van Doorn, A. J., \& KoenderinK, J. J. (1983). Detectability of velocity gradients in moving random-dot patterns. Vision Research, 23, 799-804.

Verstraten, F. A. J., Fredericksen, R. E., van Wezel, R. J. A., BoulTON, J. C., \& VAN DE GRIND, W. A. (1996). Directional motion sensitivity under transparent motion conditions. Vision Research, 36, 2333-2336.

Watamaniuk, S. N. J., Sekuler, R., \& Williams, D. G. (1989). Di- 
rection perception in complex dynamic displays: The integration of direction information. Vision Research, 29, 47-59.

Watanabe, T., \& Cole, R. (1995). Propagation of local motion correspondence. Vision Research, 35, 2853-2861.

WATSON, A. B., \& ECKERT, M. P. (1994). Motion-contrast sensitivityvisibility of motion gradients of various spatial frequencies. Journal of the Optical Society of America A, 11, 496-505.

Williams, D. W., \& Phillips, G. (1987). Cooperative phenomena in the perception of motion direction. Journal of the Optical Society of America A, 4, 878-885.

Williams, D. W., \& SeKuler, R. (1984). Coherent global motion percepts from stochastic local motions. Vision Research, 24, 55-62.
Wilson, H. R, \& Kim, J. (1994). A model for motion coherence and transparency. Visual Neuroscience, 11, 1205-1220.

YANG, Y. D., \& BlAKE, R. (1994). Broad tuning for spatial-frequency of neural mechanism underlying visual-perception of coherent motion. Nature, 371, 793-796.

Zohary, E., ScASE, M. O., \& Braddick, O. J. (1996). Integration across directions in dynamic random dot displays: Vector summation or winner take all? Vision Research, 36, 2321-2331.

(Manuscript received September 19, 1998; revision accepted for publication October 3, 2000.) 